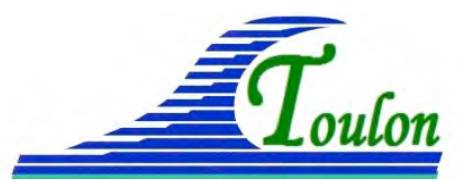

XIV vèmes Journées Nationales Génie Côtier - Génie Civil

Toulon, 29 juin au $1^{\text {er juillet } 2016}$

DOI:10.5150/jngcgc.2016.077 $\quad$ (C) Editions Paralia CFL

disponible en ligne - http://www.paralia.fr - available online

\title{
Gestion du littoral face aux risques : application de la méthode VSC
}

\author{
Isabelle LIENARD ${ }^{1}$, Cédric LEFEBVRE ${ }^{1}$
}

1. Cerema, Direction Territoriale Nord-Picardie, Sequedin, France.

isabelle.lienard@cerema.fr ; cedric.lefebvre@cerema.fr

\section{Résumé :}

La question du risque de submersion marine s’inscrit dans les réflexions générées par les changements climatiques attendus au cours des décennies à venir. Les objectifs principaux de la mission du Cerema étaient de fournir un outil de recensement, d'évaluation et de suivi des défenses (naturelles ou artificielles) contre la submersion marine dans le Nord-Pas-de-Calais. Pour cette mission, le Cerema a choisi de mettre en œuvre les principes de la méthode des "visites simplifiées comparées" (VSC). Il s’agit d'une méthode générique élaborée en 1994 par le Laboratoire Régional des Ponts et Chaussée d’Angers, dont les fondamentaux ont été adaptés depuis à de nombreuses problématiques (ports, ouvrages d'art, sentiers littoraux...).

Cette méthode offre la particularité de ne pas nécessiter un relevé détaillé des désordres, mais d'analyser les processus de dégradation du fonctionnement mécanique de l'ouvrage. La programmation optimisée des travaux à effectuer en urgence ou en entretien courant, est obtenue en croisant l'évaluation de l'état (résultat des visites techniques) et l'importance stratégique (fixée par le gestionnaire).

Pour l'évaluation technique, le patrimoine a été structuré (découpage en ouvrages et objets) et des règles d'évaluation ont été créées pour les ouvrages qui n’avaient pas encore fait l'objet d'une application de la méthode, notamment pour les ouvrages naturels comme les cordons dunaires. Comparé à d'autres patrimoines d'ouvrages, celui du littoral pose le problème de la diversité des gestionnaires et propriétaires.

Cette mission a permis aux services de l'État d'avoir une vue d'ensemble des types d’ouvrages présents le long du littoral et de leur état structurel.

Actuellement, les services de l'État continuent d'utiliser cette méthode et d'effectuer des visites annuelles. Des visites sont également programmées à la suite des événements tempétueux. Cette connaissance permet de définir les orientations pour une gestion durable du trait de côte sur le littoral.

Mots-clés : Submersion, Gestion du patrimoine, Littoral.

\section{Contexte de l'étude}

Un des objectifs des DDTM est de posséder un recensement exhaustif du patrimoine des défenses artificielles ou naturelles, contre les submersions marines de la zone NordPas-de-Calais (hors ports) et de disposer d'instruments homogènes d'évaluation et de 


\section{Thème 7 - Risques côtiers}

suivi pour assurer une gestion coordonnée de ce patrimoine. Pour atteindre cet objectif, les DDTMs ont confié au Cerema une mission (LEFEBVRE et al., 2007 et LEFEBVRE \& LIENARD, 2009) consistant à :

- définir la méthodologie de structuration du patrimoine, de découpage et d'évaluation des ouvrages en dur (perrés, digues), souples (épis) et naturels (cordons dunaires),

- tester cette méthodologie sur deux secteurs,

- former les agents des DDTMs aux visites,

- contrôler la bonne application de la méthode par des visites croisées,

- apporter une aide aux DDTMs pour l'évaluation des ouvrages complexes.

La méthode Visites Simplifiées Comparées (VSC) a été déployée sur deux zones test (voir figure 1).

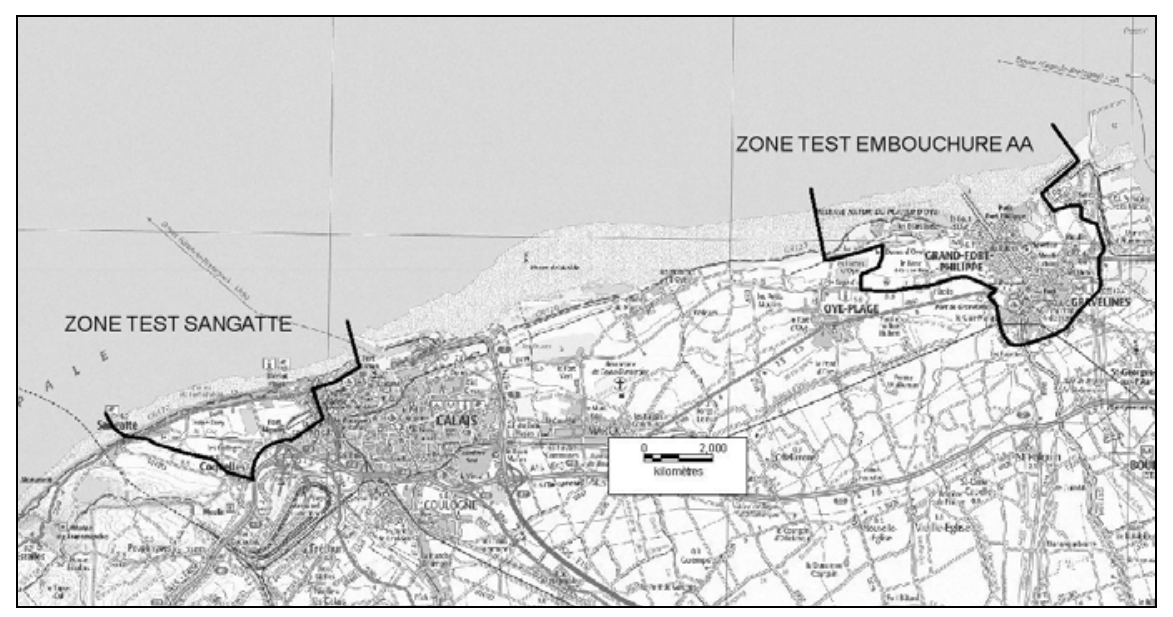

Figure 1. Localisation des deux zones test.

\subsection{Zone test de Sangatte}

Cette zone a été choisie en raison de :

- la présence de polders importants à l'arrière et d'un fort réseau de Wateringues (réseau de fossés et de pompes qui permettent l'évacuation des eaux continentales vers la mer),

- la présence de digues intérieures de seconde défense,

- la présence d'un cordon dunaire important et d'une digue en front de mer.

\subsection{Zone test de l'embouchure de l'Aa}

Le site de l'Aa a été choisi en complément de celui de Sangatte, car il présente une typologie d'ouvrages plus diversifiée. Il comporte aussi d'autres spécificités :

- l'embouchure de l'Aa et ses infrastructures (berges, barrages, écluses, vannages...),

- des dunes de part et d'autre de l'embouchure avec pour l'un, l'insertion au plus près du rivage d'un lotissement en partie protégé par des digues intérieures,

- une forte concentration urbaine et un secteur industriel sensible en limite du site (centrale nucléaire de Gravelines). 


\section{XIV èmes Journées Nationales Génie Côtier - Génie Civil \\ Toulon, 29 juin au $1^{\text {er }}$ juillet 2016}

\section{La méthode VSC}

\subsection{Concepts}

La méthode VSC est un outil d'aide à la gestion d'un patrimoine d'ouvrages, élaborée en 1994 par le Laboratoire Régional des Ponts et Chaussées d’Angers. Il répond à trois objectifs : accéder à une vue d'ensemble de l'état du patrimoine, traiter immédiatement les problèmes de sécurité, et programmer les actions curatives et préventives d'entretien. VSC consiste à évaluer les ouvrages selon trois axes indépendants (LCPC 2006) :

- un axe mécanique : évaluer la fonction mécanique de l’ouvrage,

- un axe usage : évaluer le confort et la sécurité des usagers,

- un axe stratégique : évaluer l'importance de l'ouvrage au sein du patrimoine.

Chaque axe est lié à un indice (indice d'état mécanique (IEm), indice d'état d'usages (IEu) et indice stratégique (IS)) représentés selon un schéma organisationnel "espace VSC" où les indices mécaniques et d’usages combinés constituent le plan de la gestion technique (figure 2).

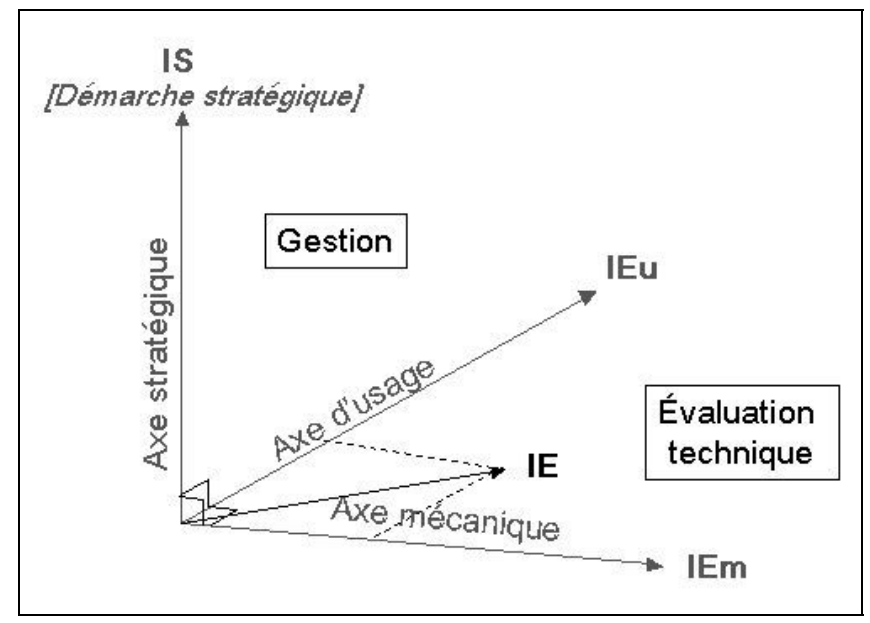

Figure 2. Principe d'évaluation des ouvrages selon trois axes.

L'évaluation des indices d'état s'appuie sur la réalisation de visites simplifiées comparées. Ces visites sont dites simplifiées car, de courte durée et ciblées sur les points critiques des structures constituants les ouvrages. Cette méthode offre donc la particularité de ne pas nécessiter un relevé détaillé des désordres mais d'analyser les processus de dégradation du fonctionnement mécanique de l'ouvrage. L'évaluation de l’indice stratégique relève de la responsabilité du gestionnaire. L'analyse croisée des différents indices permet alors de définir des priorités d'actions (surveillance, études, maintenance) nécessaires à une meilleure connaissance des ouvrages permettant l'optimisation de leur remise en état. 


\section{Thème 7 - Risques côtiers}

\subsection{Structuration}

Le déploiement de la méthode VSC s'articule autour de plusieurs étapes (THAUVIN et al., 2012). La première étape consiste à structurer le patrimoine en classant les ouvrages recensés et localisés en groupes (fonction d'utilisation générale au sein du patrimoine) et familles (fonction d'utilisation particulière au sein d'un groupe) pour avoir une meilleure lisibilité en termes de connaissance fonctionnelle et d'enjeux.

La seconde étape consiste à décomposer les ouvrages en objets pour tenir compte des structures de génie civil qui les constituent et conditionnent leur comportement mécanique et leur vieillissement. Un ouvrage peut être composé d'un ou plusieurs objets. Un objet correspond à une structure. Une structure correspond à un mode de fonctionnement mécanique et un matériau principal. Un nouvel objet est créé à chaque changement de structure ou d'utilisation particulière. L'évaluation de l'ouvrage s'effectue par l'évaluation des objets qui le composent.

La troisième étape, phase opérationnelle de la méthodologie, est la réalisation périodique de visites VSC. Ces visites portent sur l'ensemble des objets constituant chaque ouvrage et permettent d'identifier d'éventuelles anomalies sur les structures et les éléments d'usages. Une visite VSC n'est pas une inspection détaillée, ni une auscultation spécialisée. En revanche, elle pourra conclure à la nécessité d'une inspection détaillée ou d'investigations complémentaires en cas d'anomalie ou de doute sur l’intégrité de l’ouvrage.

La quatrième étape consiste à évaluer, pour chaque objet, le niveau d'indice d'état mécanique (IEm) et d'indice d'état d'usage (IEu) en s'appuyant sur une bibliothèque d'identifiants constituant une grille d'aide à la cotation. La méthode définit quatre niveaux d'indices en fonctions du degré d'endommagement, de la nature et de l'échéance de l'action à mettre en œuvre (tableau 1).

Tableau 1. Principe d'évaluation de l'état des ouvrages.

\begin{tabular}{|c|c|c|c|c|c|}
\hline & Évaluation de l'état & & Actions & & \\
\hline$I E$ & Mécanique & Usage & Niveau & Type & Échéance \\
\hline 1 & $\begin{array}{l}\text { Désordres mécaniques graves } \\
\text { Risque de ruine immédiate }\end{array}$ & $\begin{array}{l}\text { Problèmes de sécurité } \\
\text { immédiate }\end{array}$ & \multicolumn{2}{|c|}{ Mise en sécurité immédiate } & Immédiat \\
\hline 2 & $\begin{array}{l}\text { Désordres mécaniques graves } \\
\text { sans risque de ruine } \\
\text { immédiate }\end{array}$ & $\begin{array}{l}\text { Situation créant des } \\
\text { difficultés d'exploitation }\end{array}$ & $\begin{array}{l}\text { Renforcement } \\
\text { ou réparation }\end{array}$ & Curatif & $\begin{array}{l}\text { Court } \\
\text { terme }\end{array}$ \\
\hline 3 & $\begin{array}{l}\text { Dégradation des matériaux ou } \\
\text { désordres mécaniques sans } \\
\text { gravité }\end{array}$ & $\begin{array}{l}\text { Situation créant des } \\
\text { problèmes d'inconfort }\end{array}$ & $\begin{array}{l}\text { Entretien } \\
\text { spécialisé ou } \\
\text { réparation }\end{array}$ & \multirow[t]{2}{*}{ Préventif } & $\begin{array}{l}\text { Moyen } \\
\text { terme }\end{array}$ \\
\hline 4 & Bon état structurel & $\begin{array}{l}\text { Bon état des éléments } \\
\text { d'usage }\end{array}$ & $\begin{array}{l}\text { Entretien } \\
\text { courant }\end{array}$ & & $\begin{array}{l}\text { Long } \\
\text { terme }\end{array}$ \\
\hline
\end{tabular}




\section{XIV ${ }^{\text {èmes }}$ Journées Nationales Génie Côtier - Génie Civil \\ Toulon, 29 juin au $1^{\text {er }}$ juillet 2016}

La cinquième étape consiste à identifier les actions nécessaires à la remise en état des ouvrages et des objets en fonction de l'évaluation faite à l'étape précédente.

La sixième et ultime étape de la méthode consiste à définir les priorités d'intervention en croisant les indices d'état (IEm et IEu) et l'indice stratégique (IS).

\section{Application sur un patrimoine d'ouvrages du littoral en zone submersible}

\subsection{Structuration du patrimoine et décomposition des ouvrages en objets}

La méthode a été appliquée pour la première fois sur ce type d’ouvrage. Pour cette étude, les cordons dunaires sont considérés comme "ouvrages" naturels. Les "ouvrages" artificiels comportent toutes constructions de génie civil ou en terre, ponctuelles ou linéaires; soit faisant obstacle à l'eau, soit insérés dans un linéaire de défense hydraulique (portes à la mer, portes d'écluses, etc.).

Les ouvrages naturels ou artificiels présents dans la zone d'étude et impactés par la problématique de submersion ont été classés en groupes (fonction d'utilisation générale) et familles (fonction d'utilisation particulière). Certaines familles ont dû être créées, notamment pour les ouvrages "épis et cordons dunaires".

Le découpage des ouvrages en objets élémentaires est fondamental pour la future évaluation des ouvrages. Il permet en associant aux structures de ces objets un fonctionnement mécanique et un matériau principal connu et observable, d'apprécier leurs points faibles et leurs modes de dégradations privilégiés. La décomposition des ouvrages peut être transversale et/ou longitudinale (figure 3). Chaque objet est alors affecté d'une structure et d'éléments d'usages.

Par exemple, les ouvrages naturels "cordons dunaires" ont été découpés transversalement en quatre objets (de la mer vers les terres) : le haut estran, le versant amont, le corps de dune et le versant aval.

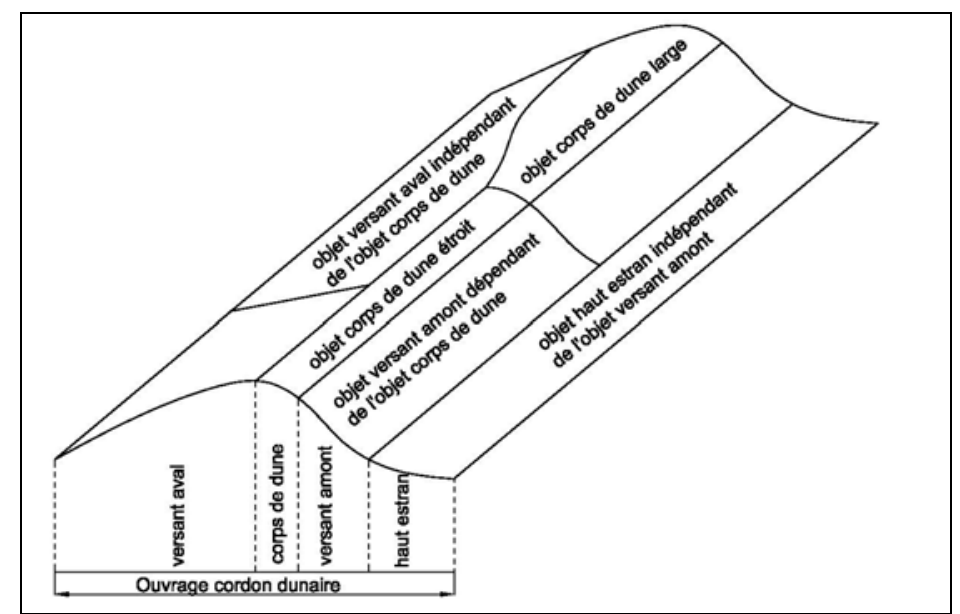

Figure 3. Exemple de décomposition d'un ouvrage cordon dunaire en objets. 


\section{Thème 7 - Risques côtiers}

Pour tous ouvrages de front de mer (artificiels et naturels), un objet "pied sur haut estran" a été ajouté, afin d'intégrer la dynamique sédimentaire en haut de plage (érosion ou engraissement) dans l'évaluation de leur état. Une baisse du niveau de sable en pied ou un affouillement a une influence sur leur stabilité.

\subsection{Nouvelles structures et règles d'évaluation}

Des ouvrages constitués essentiellement de matériaux naturels, ont été dotés de structures nouvellement crées (fonctionnement mécanique et matériau principal). Il s'agit des épis, des cordons dunaires et de certaines digues intérieures de seconde défense (tableau 2).

Tableau 2. Exemple de nouvelles structures.

\begin{tabular}{|l|l|l|l|}
\hline \multicolumn{2}{|l|}{} & \multicolumn{2}{l|}{ Structure } \\
\hline Ouvrages & Objets & Fonctionnement mécanique & Matériau principal \\
\hline \multirow{4}{*}{$\begin{array}{l}\text { Épis } \\
\text { dunaires }\end{array}$} & Ligne d'épis & Bilan sédimentaire apparent & bois \\
\cline { 2 - 5 } & Haut estran & Bilan sédimentaire apparent & Sable et enrochements ou galets \\
\cline { 2 - 5 } & $\begin{array}{l}\text { Vorpants (amont et aval) } \\
\text { large) }\end{array}$ & Talus protégé & Sable et végétation \\
\hline \multirow{2}{*}{$\begin{array}{l}\text { Digues } \\
\text { intérieures }\end{array}$} & Versants (amont ou aval) & Stock disponible & Sable et végétation \\
\cline { 2 - 5 } & Crêté et corps & Remblai perméable & Sol arboré ou végétalisé \\
\hline
\end{tabular}

Chaque structure doit disposer d'un identifiant mécanique. Ces règles d'évaluation permettent de noter l'ouvrage en répondant aux questions suivantes :

- où repérer ? Le fonctionnement mécanique de la structure induit des localisations particulières sur lesquelles les observations visuelles doivent se porter,

- que repérer ? Le matériau principal de la structure induit le type d'observations à effectuer sur son état en fonction de ses modes de dégradation,

- quand repérer ? Certains ouvrages peuvent connaître des périodes plus favorables pour l'observation des éventuels désordres à détecter.

L'objectif de ces identifiants mécaniques est de pouvoir réaliser des visites ciblées et donc relativement rapides des ouvrages tout en s'assurant que l'évaluation de l'état de l'ouvrage est objective, répétable et reproductible.

L'identifiant mécanique reprend la liste des observations susceptibles d'affecter l'ouvrage. Cette liste sert de base pour la construction des règles d'évaluation. Les observations "élémentaires" sont déclinées selon 3 ordres d'importance, faisant intervenir la vision globale de l'état (affectant la totalité de la structure d'un objet), la vision locale de l'état (affectant une partie de la structure d'un objet) et celle de l'état de dégradation du matériau de l'ouvrage (corrosion, lacune dans la maçonnerie, érosion 


\section{XIV èmes Journées Nationales Génie Côtier - Génie Civil \\ Toulon, 29 juin au $1^{\text {er }}$ juillet 2016}

des pierres...). L'absence d'observations particulières est également à noter puisqu'elle reflète un bon état de la structure et caractérise souvent un indice d'état mécanique 4.

La rédaction de l'identifiant consiste alors à prendre isolément chaque observation élémentaire décrite ci-dessus et à en définir le niveau d'indice d'état mécanique : de 1 à 4 en ordre décroissant de gravité ; et de croiser entre elles ces observations par 2, voire par 3 pour déterminer si cette combinaison est possible et si elle conduit à un niveau d’indice d'état mécanique inférieur à celui des observations élémentaires.

Pour toutes les nouvelles structures, comme le versant amont d'un cordon dunaire (voir tableaux 3, 4 et 5), qui n'avaient pas été rencontrées lors des applications VSC précédentes, des règles d’évaluation et des identifiants mécaniques ont été créés.

Tableau 3. Liste des critères d'évaluation de l'état de l'objet "versant amont".

\begin{tabular}{|l|l|l|l|}
\hline A & Affleurement du substrat & $F$ & Présence de micro-falaises en pied \\
\hline B & $\begin{array}{l}\text { Vestiges militaires déchaussés et } \\
\text { déstabilisés }\end{array}$ & G & Présence d'érosion gravitaire \\
\hline C & Résurgence de la nappe & $H$ & Présence d'érosion éolienne en crête (siffle-vent) \\
\hline D & Fascines, ganivelles, clôtures ensablées & I & Absence de signe caractéristiques \\
\hline E & $\begin{array}{l}\text { Fascines, ganivelles, clôtures } \\
\text { déchaussées (découvertes) }\end{array}$ & $J$ & $\begin{array}{l}\text { Cordon dunaire étroit (largeur équivalente à au } \\
\text { moins 2x l'érosion maxi provoquée par une tempête) }\end{array}$ \\
\hline
\end{tabular}

Tableau 4. Croisement des observations de l'objet "versant amont".

\begin{tabular}{lllllllllll}
\hline & $A$ & $B$ & $C$ & $D$ & $E$ & $F$ & $G$ & $H$ & $I$ & $J$ \\
\hline$A$ & 2 & & & & & & & & & \\
$B$ & 2 & 3 & & & & & & & & \\
$C$ & 2 & 2 & 2 & & & & & & & \\
$D$ & & 4 & & 4 & & & & & & \\
$E$ & 2 & 2 & 2 & & 2 & & & & & \\
$F$ & 2 & 2 & 2 & 3 & 2 & 2 & & & & \\
$G$ & 2 & 2 & 2 & 3 & 2 & 2 & 2 & & & \\
$H$ & 2 & 2 & 2 & & 2 & 2 & & 2 & & \\
$I$ & & & & & & & & & 3 & \\
$J$ & 1 & 2 & 1 & 3 & 1 & 1 & 1 & 1 & 2 & \\
\hline
\end{tabular}

\subsection{Niveau d'indice d'état}

\section{a) Indice d'état mécanique}

L'évaluation de l'ouvrage s'effectue par l'évaluation des objets qui le composent. L'ouvrage prendra la note la plus défavorable de l'ensemble de ses objets.

En fonction des constatations effectuées lors de la visite VSC et du niveau de l'indice d'état mécanique, des actions sont proposées pour ramener la structure à un indice plus favorable. 


\section{Thème 7 - Risques côtiers}

Un objet versant amont d'un ouvrage cordon dunaire peut avoir un IEm=1 : exemple du cordon de Grand Fort Philippe au droit du lotissement des Escardines. En raison d'un "faible stock" du corps de dune associé à la présence de micro-falaises en pied et d'érosion gravitaire et éolienne en crête du versant. L'action à court terme sur cet ouvrage naturel relève essentiellement de la surveillance avant la mise en place de pièges à sable en pied de dune. En cas de prévision de tempête, les services de l'État ont connaissance de la localisation des "points faibles" de la façade vis-à-vis de la problématique submersion marine et donc des ouvrages à surveiller en priorité.

L’objet épi, de l'ouvrage épi en pieux bois, ne peut pas avoir d’IEm de 1, car ce dispositif qui favorise la sédimentation influe sur le long terme. L’IEm de 1 désigne des objets dont les désordres pourraient avoir des conséquences immédiates. La ruine de la ligne d'épi n’impacte pas directement la prédisposition à la submersion. Pour cette structure, l’indice le plus défavorable sera un IEm de 2.

Tableau 5. Exemple d'écriture de l'identifiant de l'objet "versant amont".

\begin{tabular}{|c|c|c|}
\hline IDENTIFIANT & & \\
\hline $\begin{array}{l}\text { Repérer sur cordon dunaire étroit: } \\
\text { affleurement du substrat ou } \\
\text { résurgence de la nappe ou } \\
\text { fascines, ganivelles, clôtures déchaussées (découvertes) ou } \\
\text { présence de micro-falaises en pied ou } \\
\text { présence d'érosion gravitaire ou } \\
\text { présence d'érosion éolienne en crête (siffle-vent) }\end{array}$ & alors & 1 \\
\hline $\begin{array}{l}\text { Repérer sur cordon dunaire étroit : } \\
\text { Absence de signe caractéristique ou } \\
\text { Vestiges militaires (ou autres) déchaussés et déstabilisés } \\
\text { Repérer sur cordon dunaire large: } \\
\text { affleurement du substrat et/ou } \\
\text { résurgence de la nappe et/ou } \\
\text { Fascines, ganivelles, clôtures déchaussées (découvertes) et/ou } \\
\text { Présence de micro-falaises en pied et/ou } \\
\text { Présence d'érosion gravitaire et/ou } \\
\text { Présence d'érosion éolienne en crête (siffle-vent) }\end{array}$ & alors & 2 \\
\hline $\begin{array}{l}\text { Repérer sur cordon dunaire étroit: } \\
\text { Fascines, ganivelles, clôtures ensablées (recouvertes) } \\
\text { Repérer sur cordon dunaire large: } \\
\text { Absence de signe caractéristique ou } \\
\text { fascines, ganivelles, clôtures ensablées (recouvertes) et présence de } \\
\text { micro-falaises en pied ou } \\
\text { fascines, ganivelles, clôtures ensablées (recouvertes) et présence } \\
\text { d'érosion gravitaire ou } \\
\text { Vestiges militaires (ou autres) déchaussés et déstabilisés }\end{array}$ & alors & 3 \\
\hline $\begin{array}{l}\text { Repérer sur cordon dunaire large: } \\
\text { fascines, ganivelles, clôtures ensablées (recouvertes) }\end{array}$ & alors & 4 \\
\hline
\end{tabular}




\section{XIV èmes Journées Nationales Génie Côtier - Génie Civil \\ Toulon, 29 juin au $1^{\text {er }}$ juillet 2016}

\section{b) Indice d'état d'usage}

L’intervention sur le site n'avait pas un caractère de recensement détaillé de toutes les situations à risques en termes d'usage au sens VSC, cependant un certain nombre d'ouvrages étant déjà concernés par des problèmes de sécurité liés à l'absence ou l'endommagement des dispositifs limitants leurs accès aux publics (barrages tête d'écluse, versant de digue, etc.), ces lieux ont été sécurisés.

c) Indice d'état stratégique

L'indice stratégique permet de classer l'ouvrage suivant les priorités de gestion. Il peut prendre en compte par exemple : les enjeux financiers, politiques, les impacts socio-économiques, la sécurité publique, les conditions d'accès en cas de crise, etc. Cette réflexion peut également conduire à définir les types d'actions curatives et préventives auxquelles il est possible d'associer une estimation des coûts.

En l'absence d'un gestionnaire commun, la DDTM a décidé d'attribuer un IS en fonction de la population protégée par l'ouvrage.

\subsection{Visites croisées et appui technique}

Suite au déploiement de la méthode sur les deux zones test, la mission s'est poursuivie par la formation des agents de la DDTM à l'application de la méthode, afin qu'ils réalisent l'ensemble de l'évaluation des ouvrages du littoral. Le Cerema a accompagné les agents de la DDTM lors des premières visites et des visites croisées ont été réalisées afin de comparer les évaluations de la DDTM et du Cerema.

Le Cerema est également intervenu en appui technique pour les nouvelles structures non présentes dans les zones test qui n'avaient pas fait l'objet de création de règles d'évaluation, et pour effectuer des visites de structures complexes (portes à la mer).

\section{Conclusions}

\subsection{Les limites de l'application sur ce patrimoine}

Bien qu'adaptée au contexte, l'application de la méthode VSC à ce patrimoine d'ouvrages de lutte contre la submersion marine présente quelques limites :

- l'altimétrie de l’ouvrage n’est pas intégrée,

- pour les perrés et les digues, seule la carapace est visible et pour les ouvrages les plus anciens les coupes ne sont pas disponibles,

- en cordons dunaires, on préconise pour les ouvrages sensibles des visites en fin d'été, en fin d'hiver et suite aux événements tempétueux, afin de définir la dynamique sédimentaire du secteur,

- les actions immédiates sont souvent des réparations très ponctuelles ne concernant que le désordre. Il faudrait y associer une auscultation détaillée de l'ouvrage concerné, 


\section{Thème 7 - Risques côtiers}

- la multitude de gestionnaires pour l'ensemble des ouvrages rend difficile la programmation des actions.

\subsection{La continuité et l'utilité de cette application aux ouvrages littoraux}

Le littoral du Nord-Pas-de-Calais (hors domaine portuaire) est composé d'environ 247 ouvrages au sens de la méthode VSC : 219 artificiels (89\%) et 28 naturels (11\%).

L'exploitation des visites VSC a permis :

- de mettre en évidence 6 sites prioritaires sur le littoral du Pas-de-Calais soumis à une forte érosion et/ou à l'aléa submersion marine,

- la réalisation de réparations immédiates (Gravelines et Sangatte),

- le lancement d'études spécifiques en vue de réparations plus lourdes (Le Portel, Sangatte et Dunkerque).

Le recensement exhaustif et les évaluations des ouvrages effectués dans le cadre de l'application de la méthode VSC sont maintenant régulièrement utilisés :

- PPR littoral pour la définition des brèches potentielles, 2011,

- étude de dangers de la digue de Sangatte, 2013,

- diagnostic et élaboration d'orientation pour une gestion durable du trait de côte sur le littoral de la côte d'Opale (phase 1-État des connaissances), 2014,

- stratégie départementale de gestion du domaine public maritime naturel, 2014,

- actualisation des atlas de sensibilité POLMAR Terre (géomorphologie), à paraître,

- actualisation du catalogue sédimentologique des côtes françaises - Fascicule MancheMer du Nord, à paraître.

La DDTM62 effectue annuellement les visites des ouvrages du littoral du Nord-Pas-deCalais soumis à l'aléa submersion. Des visites complémentaires sont programmées suite aux événements tempétueux. La méthode VSC est également utilisée par les trois grands ports de la région pour la gestion de leurs ouvrages : le port de Calais depuis 2003, le port de Boulogne depuis 2005 et le Grand Port Maritime de Dunkerque depuis 2007.

\section{Références bibliographiques}

LCPC (2006). VSC Méthode d'aide à la gestion de patrimoines. Guide technique.

LEFEBVRE C., LIENARD I. (2009). Application de la méthode VSC aux ouvrages de lutte contre la submersion marine - Zone de Sangatte, Rapport d'étude CETE NP.

LEFEBVRE C., LE GARS (LIENARD) I., MICHEL M., SALOME M, (2007). Application de la méthode VSC à la gestion d'un patrimoine d'ouvrages naturels et d'infrastructures littorales - Zone de l'Aa. Rapport d'étude CETE NP.

THAUVIN B., MARCOTTE C., ROUXEL N., BOURNETON N. (2012). Application de la méthode VSC pour la gestion d'un parc d'infrastructures portuaires et maritimes. Journées Nationales Génie Côtier - Génie Civil, Cherbourg, pp 799-808. http://dx.doi.org/10.5150/ingcgc.2012.087-T 